\title{
LES of $n$-Dodecane Spray Combustion Using a Multiple Representative Interactive Flamelets Model
}

\author{
Marco Davidovic ${ }^{1 *}$, Tobias Falkenstein ${ }^{1 *}$, Mathis Bode ${ }^{1}$, Liming Cai ${ }^{1}$, Seongwon Kang ${ }^{2}$, \\ Jörn Hinrichs ${ }^{1}$ and Heinz Pitsch ${ }^{1}$ \\ ${ }^{1}$ Institute for Combustion Technology, RWTH University, 52056 Aachen - Germany \\ 2 Department of Mechanical Engineering, Sogang University, Mapo, Seoul 121-742 - Korea \\ e-mail: m.davidovic@itv.rwth-aachen.de - t.falkenstein@itv.rwth-aachen.de - m.bode@itv.rwth-aachen.de - l.cai@itv.rwth-aachen.de - \\ skang@sogang.ac.kr - j.hinrichs@itv.rwth-aachen.de - h.pitsch@itv.rwth-aachen.de \\ * Corresponding authors
}

\begin{abstract}
A single-hole n-dodecane spray flame is studied in a Large-Eddy Simulation (LES) framework under Diesel-relevant conditions using a Multiple Representative Interactive Flamelets (MRIF) combustion model. Diesel spray combustion is strongly affected by the mixture formation process, which is dominated by several physical processes such as the flow within the injector, break-up of the liquid fuel jet, evaporation and turbulent mixing with the surrounding gas. While the effects of nozzle-internal flow and primary breakup are captured within tuned model parameters in traditional Lagrangian spray models, an alternative approach is applied in this study, where the initial droplet conditions and primary fuel jet breakup are modeled based on results from highly resolved multiphase simulations with resolved interface. A highly reduced chemical mechanism consisting of 57 species and 217 reactions has been developed for $\mathrm{n}$-dodecane achiving a good computational performance at solving the chemical reactions. The MRIF model, which has demonstrated its capability of capturing combustion and pollutant formation under typical Diesel conditions in Reynolds-Averaged Navier-Stokes (RANS) simulations is extended for the application in LES. In the standard RIF combustion model, representative chemistry conditioned on mixture fraction is solved interactively with the flow. Subfilter-scale mixing is modeled by the scalar dissipation rate. While the standard RIF model only includes temporal changes of the scalar dissipation rate, the spatial distribution can be accounted for by extending the model to multiple flamelets, which also enables the possibility of capturing different fuel residence times. Overall, the model shows good agreement with experimental data regarding both, low and high temperature combustion characteristics. It is shown that the ignition process and pollutant formation are affected by turbulent mixing. First, a cool flame is initiated at approximately stoichiometric mixture and propagates towards the rich side. Hence, heat and radicals are transported away from the most reactive mixture and thus the ignition is delayed. At the same time, the transported heat and radicals increase the reactivity of rich mixtures, which strongly affects the CO formation. NO was found to increase compared to the no transport case due to enhanced mixing, which is related to a broader high-temperature zone and the additional transport of oxygen from lean into high-temperature regions.
\end{abstract}




\section{INTRODUCTION}

A large fraction of today's energy consumption is attributable to the transportation sector, which is expected to grow further in the foreseeable future. While substantial effort is being directed towards electrified propulsion systems, internal combustion engines are expected to provide the majority of this transportation energy demand [1]. Therefore, further development of internal combustion engines has a huge potential to reduce environmental impact through emission reduction. However, the engine combustion process is not fully understood and thus truly predictive models do not yet exist. The Engine Combustion Network (ECN) [2] is an international collaboration of research groups that aims to advance the scientific understanding of combustion at engine relevant conditions. In particular, the combustion process of a single-hole Diesel injector using $n$-dodecane, denoted as "Spray A", has been a principal focus. In the present work, the Spray A target case, which is defined as a 1,500 bar single injection at $900 \mathrm{~K}$ gas temperature and 60 bar back-pressure is studied. Even though significant progress has been made in experimental methods, only limited information of the in-cylinder combustion process can be extracted. Computational tools can provide additional insight in order to exploit the full potential of internal combustion engines regarding emission reduction and efficiency gain. Large-Eddy Simulations (LES) solve a filtered flow field and thus resolve the large scale turbulent fluctuations. Hence, LES describe the mixing fields more accurately compared to the classical Reynolds-Averaged Navier-Stokes (RANS) approach. Additionally, intrinsic cycle-to-cycle variations can be captured in LES, while RANS is a deterministic method by definition and thus only can capture cyclic variations that arise from external parameters (e.g., varying boundary conditions). The effect of the unresolved scales on the solution needs to be modeled in both approaches. Especially in reactive flows, where chemical reactions take place on very small time and length scales, an appropriate turbulence-chemistry interaction model is desirable. The most commonly applied types of turbulent combustion methods are Probability Density Function (PDF) transport equation models [3], Conditional Moment Closure (CMC) model [4], and flamelet models [5]. Transported PDF models solve a joint PDF depending on temperature, pressure, and reacting species for evaluating the filtered chemical source term. While further closure of the chemical source term can be avoided, additional modeling needs to be introduced regarding the molecular mixing terms. CMC models assume that scalar quantities and their fluctuations are directly correlated to mixture fraction and its fluctation, respectively. Following this assumption, transport equations can be derived for on mixture fraction conditioned reactive scalars. The chemical source term is then evaluated based on the conditionally averaged quantities. The flamelet formulation views a turbulent flame as an ensemble of multiple laminar flamelets, which can be decoupled from the turbulent flow. The chemistry for unsteady non-premixed combustion can be formulated in mixture fraction space according to:

$$
\begin{gathered}
\rho \frac{\partial Y_{\alpha}}{\partial t}=\rho \frac{\chi}{2} \frac{\partial^{2} Y_{\alpha}}{\partial Z^{2}}+\rho \dot{\omega}_{\alpha} \\
\rho \frac{\partial T}{\partial t}=\rho \frac{\chi}{2} \frac{\partial^{2} T}{\partial Z^{2}}+\frac{\rho}{c_{\mathrm{p}}} \frac{\chi}{2}\left(\frac{\partial c_{\mathrm{p}}}{\partial Z}+\sum_{\alpha} c_{\mathrm{p}, \alpha} \frac{\partial Y_{\alpha}}{\partial Z}\right) \\
+\frac{\rho}{c_{\mathrm{p}}} \sum_{\alpha} h_{\alpha} \dot{\omega}_{\alpha}+\frac{1}{c_{\mathrm{p}}} \frac{\partial P}{\partial t}
\end{gathered}
$$

where $\rho$ is the density, $Y_{\alpha}$ is the mass fraction of species $\alpha, t$ is the time, $Z$ is the mixture fraction, $\chi$ is the scalar dissipation rate, $\dot{\omega}_{\alpha}$ is the chemical source term, $c_{\mathrm{p}}$ is the specific heat, $h_{\alpha}$ is the specific enthalpy of species $\alpha$, and $P$ is the pressure. Turbulent mixing is considered in the scalar dissipation rate, which needs to be modeled.

The Representative Interactive Flamelet (RIF) $[6,7]$ model, in which the non-premixed unsteady flamelet equations are solved interactively to the flow by considering temporal changes in pressure and scalar dissipation rate, has been developed for Diesel engine simulations. Since the scalar dissipation rate is not inherently correlated with the mixture fraction, conditional averaging from physical into mixture fraction space is required. Furthermore, by beginning the chemistry computation at Start Of Injection (SOI), different fuel residence times are not captured. Those two simplifications result in a systematical overprediction of ignition delay and burn out rate. However, both effects can be reduced significantly by increasing the number of flamelets. Barths et al. [8] extended the model towards multiple flamelets by splitting the flamelet according to spatially varying scalar dissipation rate values. It was shown that the Multiple Representative Interactive Flamelets (MRIF) model improves the ignition delay prediction significantly. A slightly different multiple flamelet approach was suggested by D'Errico et al. [9]. Here, different fuel residence times were considered by solving marker variables in the flow field that track individual portions of the fuel. D'Errico compared the performance of the MRIF model with laminar chemistry in a RANS flow solver under Spray A conditions. Even though the ignition delay was only marginally affected by turbulence effects, the laminar chemistry model failed to predict other flame characteristics like flame lift-off and heat-release, while the MRIF model showed overall good agreement with experimental results. Recently, a few LES studies of the Spray A case have been published [10-12]. 
Pei et al. [10] performed simulations with laminar chemistry showing that LES capture the experimentally observed behavior more accurately compared to RANS. However, even though the ignition delay was predicted fairly well by the LES, the flame lift-off length was significantly overpredicted. Blomberg et al. [11] investigated split injections of the Spray A case using a CMC model with LES and RANS. While flame structures, ignition delays, and flame lift-off could be well predicted by both turbulence models, the LES captured the mixing field more accurately and showed more distinct combustion recession compared to RANS. Wehrfritz et al. [12] used the Flamelet Generated Manifold (FGM) model, which has been applied successfully to $n$-heptane spray LES before [13], and compared the performance of different chemical $n$-dodecane mechanisms. Besides the overprediction of the flame lift-off length for high ambient oxygen concentrations, the model showed overall good agreement with experimental data. In the FGM model, precomputed chemistry is parameterized over mixture fraction, strain rate, and progress variable. However, the progress variable cannot be defined universally and some knowledge of the combustion progress has to be incorporated into its formulation. While the state of high-temperature ignition can be well characterized by the major combustion products, the low temperature combustion is rather denoted by intermediate species. Thus, Wehrfritz et al. [12] used a linear combination of $\mathrm{CO}, \mathrm{CO}_{2}$, and $\mathrm{CH}_{2} \mathrm{O}$, where $\mathrm{CH}_{2} \mathrm{O}$ can be attributed to the first stage and $\mathrm{CO}$ and $\mathrm{CO}_{2}$ to the second stage of ignition, respectively. Since the chemistry is computed under constant strain rate, the effect of its temporal evolution is not captured correctly in this model. In contrast to the FGM model, the MRIF model does not rely on a predefined progress variable and is able to consider the temporal evolution of the scalar dissipation rate and pressure, which makes it particularly appealing for full engine applications. However, even though its potential of capturing spray combustion characteristics under typical Diesel conditions has been demonstrated succesfully in RANS simulations [8,9], the MRIF model has not been applied to LES studies of Diesel spray combustion yet. Hence, an LES-MRIF combustion model is presented and applied to the Spray A case in this study.

\section{SIMULATION FRAMEWORK}

\subsection{Governing Equations}

The flow is governed by the balance of mass and momentum, which is given for continuous fluids by the NavierStokes equations:

$$
\frac{\partial \rho}{\partial t}+\frac{\partial \rho u_{j}}{\partial x_{j}}=\dot{S}_{m}
$$

$$
\frac{\partial \rho u_{i}}{\partial t}+\frac{\partial \rho u_{j} u_{i}}{\partial x_{j}}=-\frac{\partial P}{\partial x_{i}}+\frac{\partial \tau_{i j}}{\partial x_{j}}+\dot{S}_{u_{i}}
$$

where $u_{j}$ is the velocity in direction $j, \tau_{i j}$ is the stress tensor, and $x_{j}$ is the spatial coordinate in the direction of $j . \dot{S}_{m}$ and $\dot{S}_{u_{i}}$ contain the volumetric mass and momentum sources, respectively. For compressible flows, the conservation of energy also needs to be solved. In this study, the internal energy formulation is used, which reads:

$$
\frac{\partial \rho e}{\partial t}+\frac{\partial \rho u_{j} e}{\partial x_{j}}=-P \frac{\partial u_{j}}{\partial x_{j}}+\tau_{i j} \frac{\partial u_{i}}{\partial x_{j}}+\frac{\partial}{\partial x_{j}}\left(\lambda \frac{\partial T}{\partial x_{j}}\right)-\frac{\partial q \prime \prime}{\partial x_{j}}+\dot{S}_{e}
$$

where $e$ is the internal energy, $\lambda$ is the thermal conductivity, $T$ is the temperature, $q^{\prime \prime}$ is the enthalpy flux caused by mass diffusion, and $\dot{S}_{e}$ includes all volumetric energy sources or sinks. An equation of state is needed to close the system of equations. In this study, the ideal gas law is used:

$$
P=\frac{\rho \mathscr{R} T}{W}
$$

where $\mathscr{R}$ is the universal gas constant and $W$ the molecular weight of the gaseous mixture.

Internal combustion engines are typically operated at high Reynolds numbers yielding extremely small turbulent structures. Resolving all length and time scales is not feasible due to the computational effort. Thus, even though the turbulent flow is completely described by the governing equations, further modeling needs to be introduced. In LES, a spatial low-pass filter is applied to the flow field. Thus, large turbulent structures are retained in the solution, while small scales are damped out. Filtering the governing equations yields unclosed terms, which include the effect of the unresolved scales on the filtered solution. Those terms are modeled by the Sub Grid Scale (SGS) model. In this study, a dynamic Smagorinsky-type model [14] is used. The filter size is determined by the local mesh size and the averaging is performed over Lagrangian trajectories backward in time.

\subsection{Spray Model}

For the simulation of multiphase flows, such as engine fuel injection, two approaches are commonly used for treating the liquid phase. In interface resolving simulations, the governing equations are solved on a spatially discretized numerical grid for both, liquid and gaseous phase, and typically a special interface treatment is applied. Hence, the geometries of liquid structures are resolved and interfacial interactions can be modeled very accurately. However, engine fuel injections typically involve a wide range of length and time scales, which restricts the applicability of such models due to limited computational resources. In contrast, the Lagrangian 
formulation treats the entire liquid as discrete droplets. The droplet shape is presumed and hence the geometry does not need to be resolved on a spatial grid, hence, reducing the computational effort significantly. Interactions with the continuous gas phase are handled by volumetric source terms in Equations (3)-(5). However, since the geometrical information is lost, interfacial forces and fluxes need to be modeled by more rudimentary models, which typically include nonuniversal model parameters. Hence, Lagrangian spray models are usually only valid in a limited range of operating conditions. Especially, the initial droplet sizes and breakup model parameters vary significantly in the literature $[15,16]$.

In the present study, an alternative approach for simulating fuel sprays [17] is used, which separates the injection process into three individual simulations that are one-way coupled at the interfaces. In the farfield, a Lagrangian Spray model is used for the liquid phase in combination with an LES (LSLES) description of the gas phase. The Primary Breakup is modeled based on results of a multiphase DNS (PBDNS) with resolved interface in the vicinity of the nozzle that was coupled to an LES of the Nozzle Internal Flow (NIFLES). For more information on NIFLES, PBDNS, and their coupling, the reader is referred to [18].

The Lagrangian spray model uses a BLOB method for initializing the droplets at the nozzle orifice. The mass flow rate is taken from post-processed rate measurements, which are provided by the ECN [2]. The initial velocity magnitude is calculated from the mass flow rate, fuel density, measured nozzle diameter and area contraction coefficient. Statistics of the droplet properties have been recorded in the PBDNS at a distance of $1 \mathrm{~mm}$ from the nozzle orifice. The initial droplet velocity direction is computed from the recorded radial fuel mass distribution. After the droplet passed the PBDNS measurement location, breakup is enforced into the recorded droplet size distribution. The number of child droplets is calculated from mass conservation. The child parcels are randomly distributed within the volume of their parent after the breakup event. Further breakup models have not been applied in this study, since no major effect had been seen in previous simulations. The momentum exchange was modeled by a high velocity drag formulation for spherical droplets [15], while the evaporation model by Miller et al. [19] is employed for computing the heat and mass transfer.

\subsection{Combustion Model}

The MRIF model in a formulation similar to [9] is used in this study. A schematic describing the model is given in Figure 1. The chemistry is parameterized over mixture fraction, for which the scalar transport equation

$$
\frac{\partial \bar{\rho} \tilde{Z}}{\partial t}+\frac{\partial \bar{\rho} \tilde{u}_{j} \tilde{Z}}{\partial x_{j}}=\frac{\partial}{\partial x_{j}}\left[\bar{\rho}\left(D_{Z, t}+D\right) \frac{\partial \tilde{Z}}{\partial x_{j}}\right]+\overline{\dot{S}_{Z}}
$$

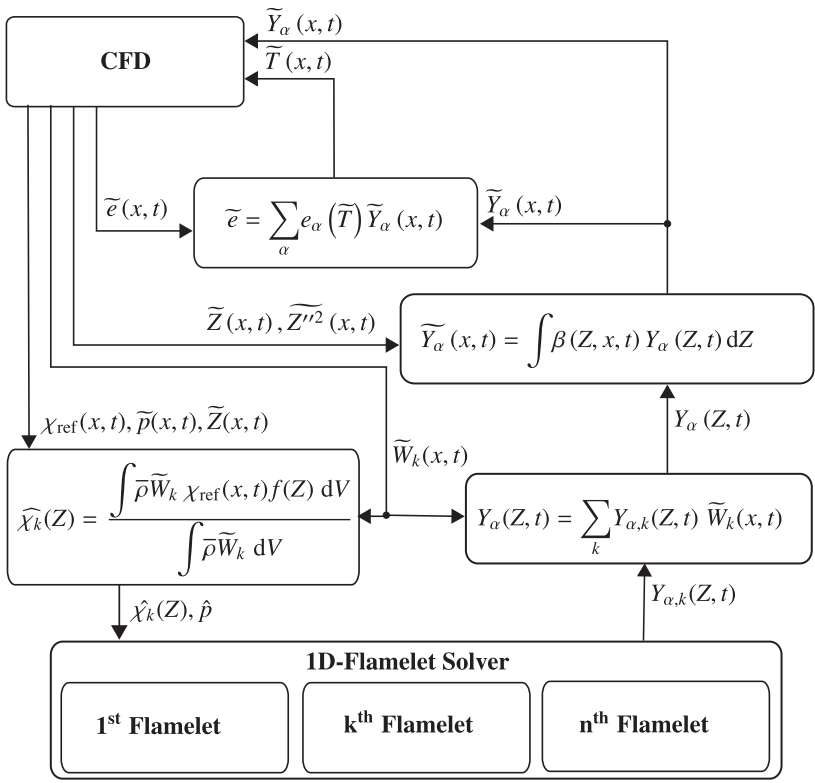

Figure 1

Schematic of the MRIF combustion model.

is solved, where $\overline{\dot{S}_{Z}}$ is the source term due to fuel evaporation, while $D$ and $D_{Z, t}$ are the molecular and turbulent diffusivity, respectively. The subfilter distribution is approximated by a beta function, $\beta$, which is parameterized with the first two filtered moments. The second moment, $\widetilde{Z^{\prime \prime} 2}$ is evaluated from an additional scalar transport equation [20] given by:

$$
\frac{\partial \bar{\rho} \widetilde{Z^{\prime \prime} 2}}{\partial t}+\frac{\partial \bar{\rho} \widetilde{u_{j}} \widetilde{Z^{\prime \prime 2}}}{\partial x_{j}}=\frac{\partial}{\partial x_{j}}\left[\bar{\rho}\left(D_{Z^{\prime \prime 2}, t}+D\right) \frac{\partial \widetilde{Z^{\prime \prime 2}}}{\partial x_{j}}\right]-\bar{\rho} \widetilde{\chi}
$$

The flamelet computation requires the spatially averaged pressure value and the scalar dissipation rate in mixture fraction space. The latter is modeled by assuming a functional form, $f(Z)$, that is scaled by a reference value, $\chi_{\text {ref }}$. The functional form can be either based on the inverse error function or logarithmic profiles. In this study, the logarithmic formulation is used and the reference mixture fraction is taken as the filtered cell value as proposed by Doran [21] as:

$$
f(Z)=\frac{Z^{2} \log \left(Z / Z_{\max }\right)}{\widetilde{Z}{ }^{2} \log \left(\widetilde{Z} / Z_{\max }\right)}
$$

In LES, both, the resolved and unresolved scales contribute to the scalar dissipation rate. In this study, it is modeled by the turbulent and molecular diffusivity and the gradient of the filtered mixture fraction [22] as:

$$
\tilde{\chi}=2\left(D_{Z, t}+D\right)\left(\frac{\partial \tilde{Z}}{\partial x_{j}}\right)^{2}
$$


The reference scalar dissipation rate can then be computed by:

$$
\chi_{\mathrm{ref}}=\frac{\tilde{\chi}}{\int f(Z) \beta(Z) \mathrm{d} Z}
$$

In spray combustion, the scalar dissipation rate evolves in time from high values at the nozzle orifice towards low values farther downstream. However, this evolution cannot be captured in a single flamelet model, since the spatial information is lost due to conditional averaging into mixture fraction space. For the multiple flamelet model, the injected fuel is splitted into $n$ portions, which are attributed to individual time intervals. The fuel portions are tracked by $n-1$ additional scalar transport equations, $M_{k}$, in the flow domain by the equation:

$$
\frac{\partial \bar{\rho} \widetilde{M_{k}}}{\partial t}+\frac{\partial \bar{\rho} \tilde{u_{j}} \widetilde{M_{k}}}{\partial x_{j}}=\frac{\partial}{\partial x_{j}}\left[\bar{\rho}\left(D_{M_{k}, t}+D\right) \frac{\partial \widetilde{M_{k}}}{\partial x_{j}}\right]+\overline{\dot{S}_{M_{k}}}
$$

Here, $\bar{S}_{M_{k}}$ is again the evaporation source term, which is equal to $\dot{S}_{Z}$ within the time interval $k$ and zero at all other times. The marker value of the last flamelet can then be computed by an analytical relation arising from mass conservation:

$$
\widetilde{Z}=\sum_{k} \widetilde{M_{k}}
$$

Furthermore, the turbulent diffusivities of the tracking scalars are determined as $D_{M_{k}, t}=D_{Z, t}$. Based on the marker scalars, weighting factors can be defined that determine the spatial distribution of the flamelets by:

$$
\widetilde{W_{k}}=\frac{\widetilde{M_{k}}}{\widetilde{Z}}
$$

By considering those weighting scalars within the conditional averaging, the spatially varying nature of the scalar dissipation rate can be retained. Furthermore, the weighting factors are used for combining the species solutions of all flamelets to the local cell values by linear combination. The flamelet computations are started at the beginning of its corresponding marker injection interval in order to capture different fuel residence times. The filtered species mass fractions are obtained by convoluting the laminar solution with the mixture fraction sub filter distribution. The filtered temperature is then iterated from the transported energy and filtered species composition. It should be noted that the flamelets are computed separately and do not exchange additionally information. Thus, mixing effects of subsequently injected fuel is only considered through the linear combination of flamelet solutions, but not in the chemistry calculation itself. Hence, if the combustion process is strongly affected by reaction products of previously injected fuel (e.g., in split injection cases), further model extensions might be required.

\subsection{Numerical Framework}

The simulations were performed with the in-house code CIAO, which is a structured, high-order, finite difference code that features both, a fully compressible and a low-Mach number solver. The flow variables are staggered in space allowing higher discretization accuracy for a given stencil size. The compressible solver was applied for all simulations in this study. It employs an explicit five-stage Runge-Kutta integration scheme in time. The momentum equations are discretized by central differences resulting in low numerical dissipation. All scalars are discretized by Weighted Essentially NonOscillatory (WENO) schemes ensuring bounded solutions [23]. A more detailed description of the flow solver is given in Desjardins et al. [24] and Mittal et al. [25].

The droplet equations are advanced prior to the gas phase in a frozen flow field using a 2 nd order Runge-Kutta scheme with adaptive time-stepping. The statistical parcel method [26], which pools droplets of similar quantities into so-called parcels, was used to reduce the computational effort. The source terms in the gas phase equations arising from the liquid droplets are distributed using a Gaussian distribution kernel [27] reducing mesh dependence and increasing numerical stability. An in-house combustion library was used as flamelet solver, which is parallelized in mixture fraction space. The flamelet equations are advanced after the flow field integration. The scalar dissipation rate is linearly interpolated in time when solving the flamelet. All flamelets were distributed equally to the total number of processors and solved simultaneously in order to minimize load imbalance.

\section{SIMULATION SETUP}

\subsection{Simulation Cases}

The simulation cases correspond to experimental studies that were conducted in a constant volume pre-burn spray chamber at Sandia National Laboratories. The experimental data is provided via the ECN data platform [2]. A detailed description of the experimental setup can be found in [28-30]. The experimental initial and boundary conditions are summarized in Table 1.

\subsection{Numerical Setup}

Both simulations were performed on the same numerical grid with a minimum grid spacing of $60 \mu \mathrm{m}$ at the nozzle 
TABLE 1

Experimental boundary and initial conditions.

\begin{tabular}{c|c|c}
\hline & Inert & Reactive \\
\hline Ambient temperature $[\mathrm{K}]$ & 892 & 903.9 \\
\hline Ambient density $\left[\mathrm{kg} / \mathrm{m}^{3}\right]$ & 22.99 & 22.92 \\
\hline Ambient pressure $[\mathrm{M} \mathrm{Pa}]$ & 6.05 & 5.98 \\
\hline $\mathrm{O}_{2}$ mole fraction $[\%]$ & 0 & 15 \\
\hline Injection pressure $[\mathrm{M} \mathrm{Pa}]$ & 150.4 & 153.5 \\
\hline Fuel temperature $[\mathrm{K}]$ & 373 & 373 \\
\hline Nozzle diameter $[\mu \mathrm{m}]$ & 83.7 & 90.8 \\
\hline Discharge coefficient $[-]$ & 0.89 & 0.89 \\
\hline
\end{tabular}

orifice and maximum grid spacing of approximately $700 \mu \mathrm{m}$ at the farthest location downstream. The geometry of the combustion vessel was approximated by a cuboid with $100 \mathrm{~mm}$ in spray axis and $60 \mathrm{~mm}$ in transverse direction, while the geometry of the injector was resolved using a stair-step approach. The acoustic Courant-Friedrichs-Lewy (CFL) number based on the entire time step was limited to 1.98 resulting in a time step of approximately $27 \mathrm{~ns}$. WENO5 has been chosen as scalar scheme, while 4th order central differences have been employed for the momentum equations. The initial parcels contained a single droplet. In order to reduce the computational cost, the number of child parcels at the primary break-up event was limited to 1,500 resulting in a total number of approximately 350,000 at quasi-steady state. The source terms arising from the liquid droplets were distributed over three cells in each spatial direction in order to stabilize the simulation. Figures $2 \mathrm{a}$ and $2 \mathrm{~b}$ show the droplet size distributions and projected fuel density profile that have been extracted from the PBDNS. A Rosin-Rammler distribution was fitted to the PBDNS results and imposed in the LSLES. The measured nozzle geometry of injector \#210675 was used in the NIFLES resulting in a nonsymmetric spray shape. Since the LSLES results were compared with experiments that have been conducted with different nozzles (\#210677 and \#210370), the projected fuel density profile has been approximated by a symmetric Gaussian distribution function (Fig. 2b) resulting in the droplet angle distribution given in Figure 2c. All initial and boundary conditions were taken from the experimental values given in Table 1. Only the pressure was computed by the equation of state according to the given density and temperature. A new flamelet and flamelet marker, $M_{k}$, was started every $60 \mu \mathrm{s}$ in the reactive case. The flamelet grid was discretized by 101 grid points with strong coarsening in very rich regions $(Z>0.4)$. The flamelet is initialized with the ambient conditions on the oxidizer side. The fuel side temperature

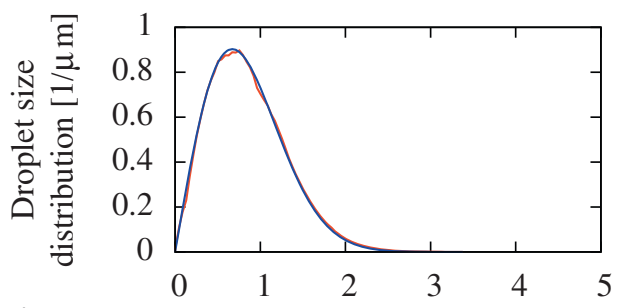

a)

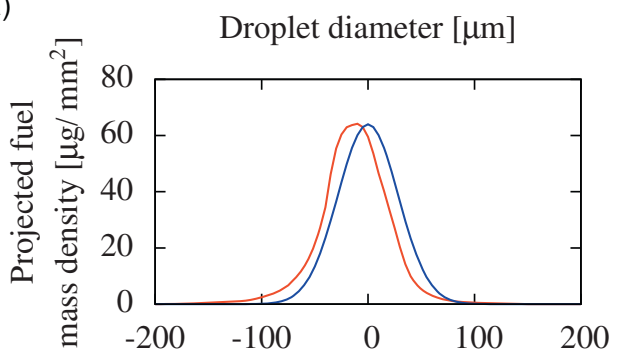

b) Transverse distance $[\mu \mathrm{m}]$

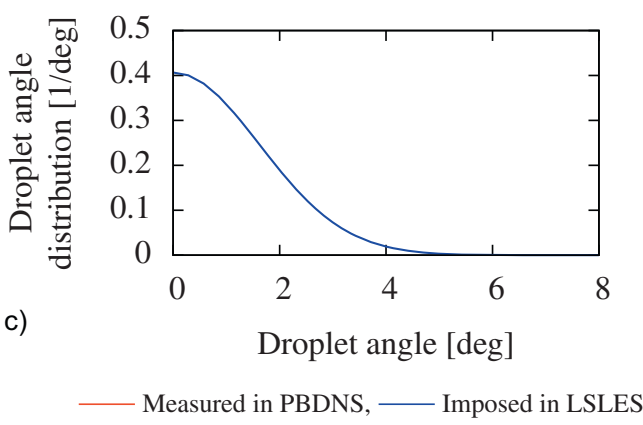

Figure 2

Primary breakup coupling quantities at $1 \mathrm{~mm}$ distance from the nozzle orifice.

is prescribed as the liquid fuel temperature corrected by an additional term that accounts for the latent heat of vaporization $[31,32]$. A summary of the numerical setup is given in Table 2.

\subsection{Chemical Mechanism}

A highly reduced reaction scheme for $n$-dodecane oxidation with an emphasis on prediction of auto-ignition process was applied in this study. The reduced model was derived from the mechanism of Narayanaswamy et al. [33], which is composed of 294 chemical species along with 2,730 reactions (forward and backward counted separately) and has been validated against the experimental measurements of ignition delay times [34]. Due to the large model size, the mechanism was reduced using a multi-stage automatic reduction strategy developed by Pepiot-Desjardins and Pitsch [35]. The Direct Relation Graph method with Error Propagation (DRGEP) was first applied to eliminate chemical species and elementary reactions from the detailed model based on the evaluation of species production and consumption rates. 
TABLE 2

Numerical setup.

\begin{tabular}{c|c}
\hline Minimum grid spacing $[\mu \mathrm{m}]$ & 60 \\
\hline Maximum grid spacing $[\mu \mathrm{m}]$ & 700 \\
\hline Grid dimensions [mm] & $100 \times 60 \times 60$ \\
\hline Number of cells [-] & $29.5 \mathrm{e} 6$ \\
\hline Number of parcels [-] & $350 \mathrm{e} 3$ \\
\hline Number of flamelets [-] & $26(1.5 \mathrm{~ms})$ \\
\hline Flamelet grid points [-] & 101 \\
\hline Time step [ns] & 27 \\
\hline
\end{tabular}

Subsequently, lumping of species isomers [36] was performed automatically, following the second species and reaction reduction process using DRGEP. The target conditions considered in the reduction process include those at which ignition delay times were reported [34]. The final reduced mechanism consists of 57 species and 217 reactions. While a strong reduction of model size was achieved, it also leads unavoidably to deteriorated model prediction accuracy. Therefore, an automatic model optimization process was applied to the reduced model for improved prediction accuracy of ignition delay times. The model optimization method developed by Frenklach [37] was employed and optimized the reaction coefficients of sensitive reactions automatically in order to give the minimum difference between model performance and experimental data. A comparison of the reduced and optimized model with the measurements reported by Vasu et al. [34] is shown in Figure 3. Over the entire investigated range, the reduced model, developed as part of this study, gives very satisfactory results. More importantly, because of the optimization process, the model also shows improved prediction accuracy against the detailed model from Narayanaswamy et al. [33], especially at low temperatures, which are of major interest for spray combustion. In order to evaluate the effect of turbulent transport on the pollutant formation, a detailed $\mathrm{NO}_{x}$ mechanism from Lamoureux et al. [38] has been coupled to the reduced $n$-dodecane mechanism. Additionally, PAH chemistry from [39] has been added to the reaction mechanism in order to enable future soot studies.

\section{RESULTS AND DISCUSSION}

\subsection{Inert Case}

The first simulation was performed under non-reactive conditions in order to demonstrate the validity of the spray
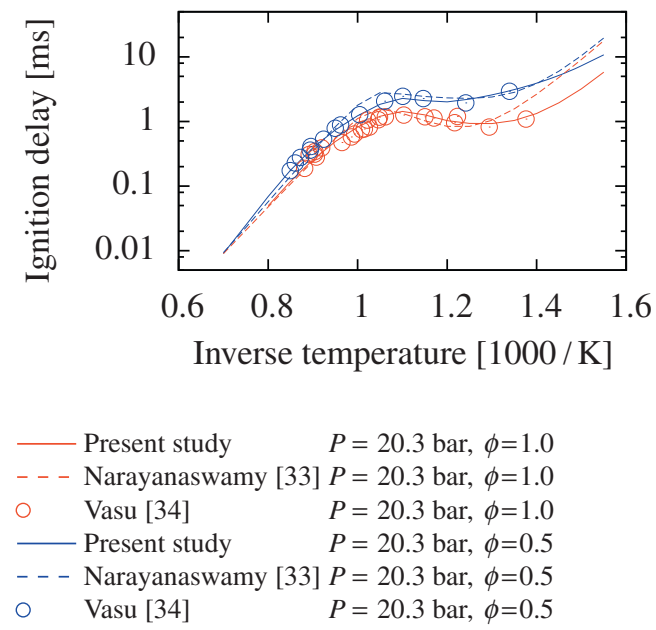

Figure 3

Comparison of baseline mechanism and reduced mechanism with experimental ignition delay measurements.

model. Figure 4 shows the LES results compared to experimental data provided by Sandia National Laboratories via the ECN online data base [2]. The vapor penetration was extracted from schlieren measurements, while the liquid penetration length was obtained by both, Diffusive Backlight Illumination (DBI) and the Mie-Scattering. The numerical values have been calculated according to the ECN modeling standards. The vapor penetration is defined as the maximum distance from the nozzle orifice to the position, where the fuel mass fraction of $0.1 \%$ is exceeded. The liquid penetration denotes the farthest axial distance from the nozzle to the position of $0.1 \%$ liquid volume fraction, which is evaluated over cylindrical volumes of $1 \mathrm{~mm}$ length and $1 \mathrm{~mm}$ diameter. It can be seen that the model predicts both, liquid and vapor penetration within the experimental uncertainties. Additionally, mixture fraction fields have been compared with experimental data. For more information about the experimental methodology, the reader is referred to [40] and [29]. The computed mixture fraction field was evaluated at two different axial positions $(17.85 \mathrm{~mm}$ and $25 \mathrm{~mm}$ ), where Favre-averaging was performed from 1-2 $\mathrm{ms}$ in time and additionally in circumferential direction. As shown in Figure 5, the model shows fairly good qualitative agreement, but underpredicts the fuel mass fraction on the spray centerline. Similar results have been reported by Wehrfritz et al. $[12,15]$ for comparable grid resolutions using Lagrangian spray models that rely on parameter tuning. In reference [15], the mixture field prediction could be improved by increasing the spatial resolution, which has not been targeted in this study due to the high computational cost. Nevertheless, considering the fact that no model parameter tuning has been performed, the overall agreement is in an acceptable range. 


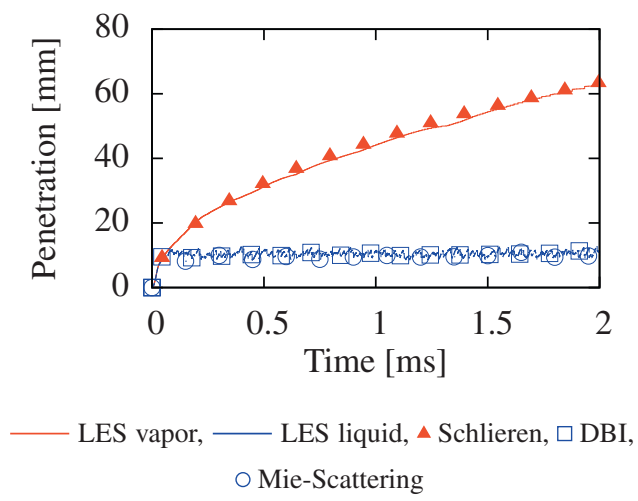

Figure 4

Temporal evolution of the spray penetration compared with experimental results [2].

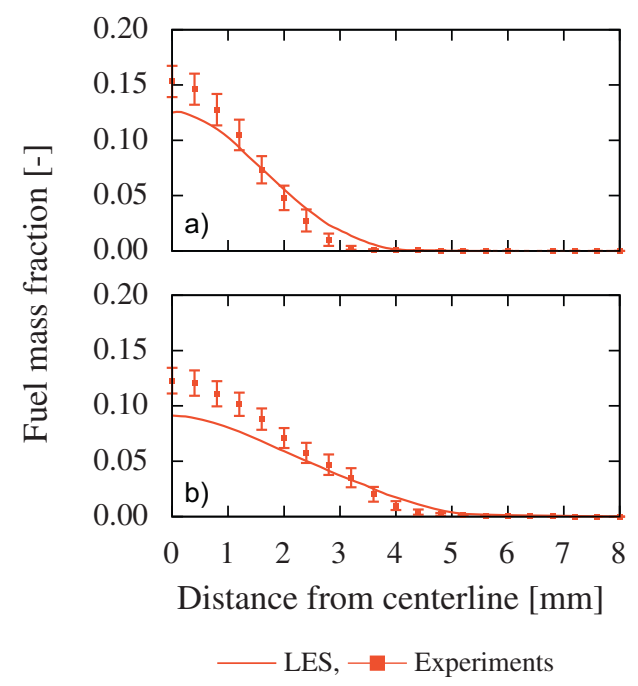

Figure 5

Favre-averaged fuel mass fraction compared with experimental results [2] at a) $17.85 \mathrm{~mm}$ and b) $25 \mathrm{~mm}$ downstream the nozzle orifice.

\subsection{Reactive Case}

\subsubsection{Low-Temperature Combustion}

The model performance is evaluated by comparison with experimental results from Skeen et al. [30], who simultaneously measured schlieren and the formaldehyde distribution. The schlieren technique visualizes gradients of the refractive index, which is directly correlated with the density, in a line-of-sight view. When the density decreases towards a value similar to the surrounding gas due to heat release from low temperature reactions, the schlieren signal softens. At that time, the spray structure is only hardly visible in the schlieren data. The schlieren signal reappears when the ignition process progresses due to additional heat release. This effect will be referred to as Schlieren-softening in the following analysis.

In order to allow a qualitative comparison of the simulation results and experimental data, representative schlieren images were extracted from the LES solution. Therefore, the absolute values of the density gradients have been integrated in transverse direction. In Figure 6, the numerical results (Fig. 6a) are plotted against the experimentally obtained images (Fig. 6b). The lower color range limit was chosen such that acoustic waves are not visible. Additionally, an isoline $(0.1 \%)$ of the maximum mixture fraction that was evaluated in line-of-sight direction is given by the red line in the numerical results in order to indicate the spray penetration.

At the very early stages $(140 \mu \mathrm{s})$, very good agreement can be observed. Both, simulation and experiment show similar spray shape and strong contrast between spray and ambient gas. Consequently, no significant heat has been released up to this point. At $260 \mu \mathrm{s}$ ASOI (After Start Of Injection), the spray penetration is slightly overpredicted by the simulation and the schlieren extracted from the LES appear a little softer in the outer spray zones. In the next three frames, stronger schlieren softening can be observed in both, experiment and simulation. At $347 \mu \mathrm{s}$, almost the complete spray head vanishes in the experimental data, which is not predicted that strongly by the model. In the last frame, both, simulation and experiment, show again stronger schlieren signal at the spray head indicating the initiation of high temperature combustion. A comparison of the formaldehyde Planar LaserInduced Fluorescence (PLIF) measurements by Skeen et al. [30] (Fig. 7a) against the LES results is given in Figure 7. The simulated $\mathrm{CH}_{2} \mathrm{O}$ mass fractions are shown on an arbitrarily chosen cut plane through the centerline (Fig. 7b) allowing a more consistent comparison with the experimental data. Additionally, the circumferential averaged values are given (Fig. 7c) in order to include more statistics. Skeen et al. [30] showed very early formation of $\mathrm{CH}_{2} \mathrm{O}$ and thus, the occurrence of low temperature chemistry prior to the high temperature ignition event. The early $\mathrm{CH}_{2} \mathrm{O}$ formation is well predicted by the model. Prior to the ignition, good agreement can be observed in both, temporal evolution and spatial distribution. However, from $340 \mu$ s on, minor discrepancies can be observed at the spray centerline behind the spray head, where the simulation underpredicts the formation of $\mathrm{CH}_{2} \mathrm{O}$. The observation is consistent with the schlieren comparison, in which the LES shows less schlieren softening in this area at the corresponding time steps. A possible explanation is the negligence of mixing effects between the individual chemistry calculations. Including those mixing effects should increase the reactivity of the subsequently injected fuel. At $490 \mu$ s, the simulation overpredicts the formaldehyde concentration in the spray head. Overall, however, the presented 

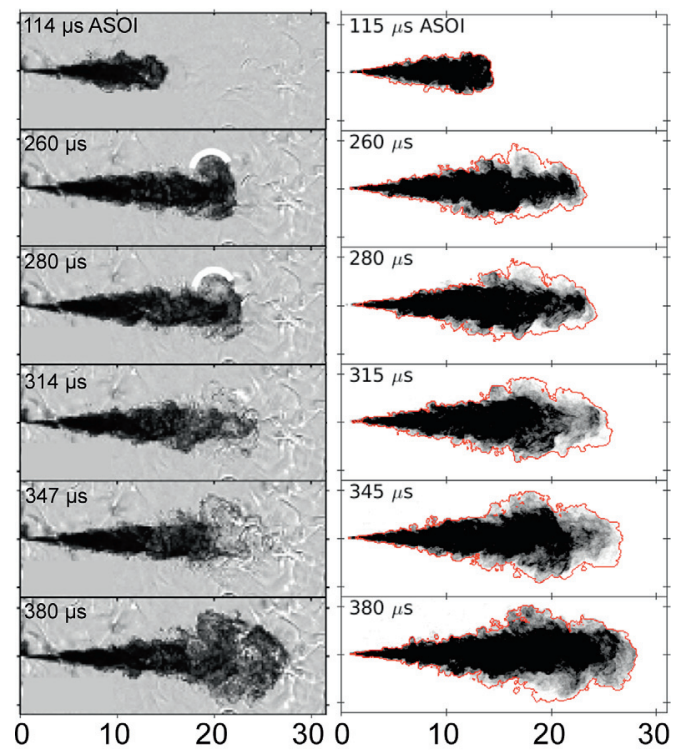

a)

b)

Distance from injector orifice [mm]

Figure 6

Schlieren images of the a) ignition process [31] against b) visualization of the predicted absolute density gradients integrated in line-of-sight direction. Reprinted from Proceedings of the Combustion Institute Volume 35, Issue 3: Scott A. Skeen, Julien Manin, Lyle M. Pickett, Simultaneous formaldehyde PLIF and high-speed Schlieren imaging for ignition visualization in high-pressure spray flames, Pages 3167-3174, 2015, with permission from Elsevier.

model agrees well with the observations by Skeen et al. [30], conforming the occurrence of low temperature combustion prior to the ignition.

\subsubsection{Ignition Delay and Flame Lift-Off}

Ignition delay and flame lift-off length directly correlate with noise and pollutant emissions in Diesel engines, and thus, need to be predicted accurately by the combustion model. Both quantities are attributed to high-temperature chemistry and can be characterized by hydroxide $(\mathrm{OH})$. The excited state $\left(\mathrm{OH}^{*}\right)$, which is formed in near-stoichiometric mixtures at high heat releases, emits light through chemiluminescence at a specific wavelength that can be measured experimentally. Hence, the ECN defines the lift-off length as the location, where $50 \%$ of the maximum intensity is reached. Accordingly, the time when $50 \%$ of the maximum is firstly exceeded is defined as ignition delay. Since $\mathrm{OH}^{*}$ is not explicitly represented in the chemical mechanism used in this work, $\mathrm{OH}$ was taken as a measure for the LES results. For the numerical studies, the ECN recommends the evaluation of the line-of-sight integrated $\mathrm{OH}$ mass fraction field with a threshold of $2 \%$ of the maximum value for both, the ignition delay and flame lift-off length. Applying the

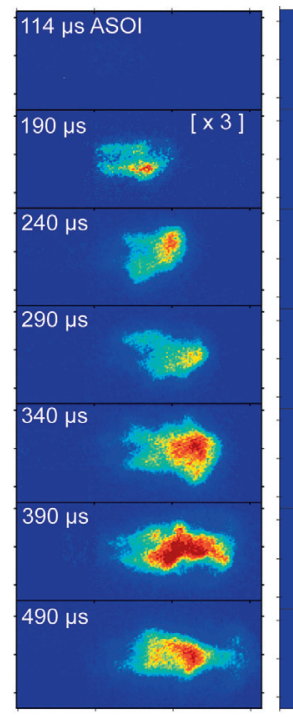

a)

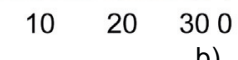

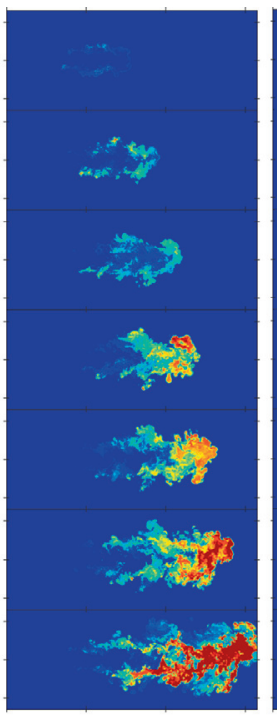

$1020 \quad 300$

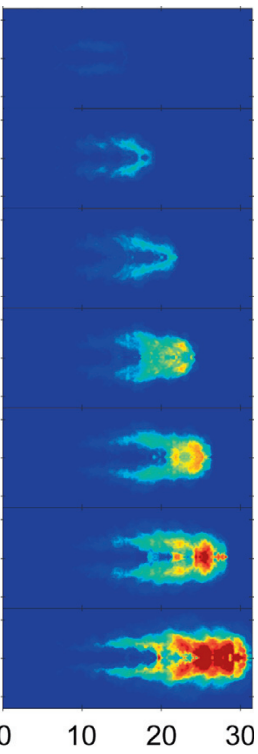

Distance from injector orifice [mm]

Figure 7

Comparison of a) $\mathrm{CH}_{2} \mathrm{O}$ PLIF measurements [31] against b) instantaneous $\mathrm{CH}_{2} \mathrm{O}$ mass fractions on a centerplane and c) circumferentially averaged $\mathrm{CH}_{2} \mathrm{O}$ mass fractions predicted by the LES. Reprinted from Proceedings of the Combustion Institute Volume 35, Issue 3: Scott A. Skeen, Julien Manin, Lyle M. Pickett, Simultaneous formaldehyde PLIF and high-speed Schlieren imaging for ignition visualization in high-pressure spray flames, Pages 3167-3174, 2015, with permission from Elsevier.

criterion to the LES results yields an ignition delay and flame lift-off of $0.435 \mathrm{~ms}$ and $13.4 \mathrm{~mm}$, respectively, while temporal averaging has been applied from 0.8 to $1.0 \mathrm{~ms}$ for the latter value. The experimental values are given by $0.4 \mathrm{~ms}$ for the ignition delay and $16.1 \mathrm{~mm}$ for the time-averaged flame lift-off length. A time sequence of the line-of-sight integrated $\mathrm{OH}$ mass fractions is given in Figure 8. The experimental time-averaged flame lift-off is additionally given by the vertical red line. At $440 \mu \mathrm{s}$, directly after ignition, strong $\mathrm{OH}$ formation is observed in multiple spots throughout the spray head. Additionally, a small flame patch occurs more closely to the injector orifice at the outer layer of the spray. In the following frames, the ignition spots in the spray head combine to a single flame structure, while some detached flames can still be observed further upstream. Those detached flame spots are mainly causing the underprediction of the flame lift-off length, while the main flame seems to be in better agreement with the experimental value. It should be noted that only a single realization was performed in the present work and ignition delay time scattered up to $0.05 \mathrm{~ms}$ in LES studies of the Spray A case with multiple realizations $[10,12]$. Overall, the simulation seems to capture the 


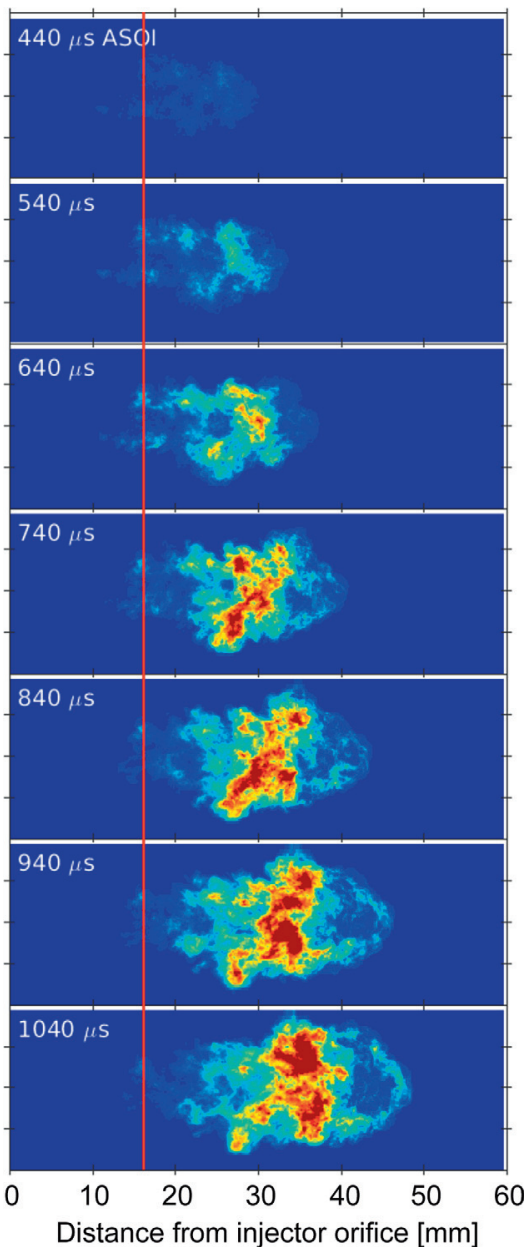

Figure 8

Time sequence of the LES OH mass fraction integrated in lineof-sight direction. The experimental time-averaged flame liftoff length is marked in red.

underlying physical processes well, since all quantities are in good agreement with the experimental findings.

\subsubsection{Ignition Process and Pollutant Formation}

Since the model show reasonable agreement with the experimental data in terms of global flame characteristics and local cool flame indicator species, the model results can be used to investigate the spray combustion process in more detail. Hence, in order to analyze the effect of turbulence-chemistry interactions, the chemistry solution of the first MRIF flamelet has been extracted from the LES and compared with a Flamelet calculation WithOut Transport (FWOT). Figure 9 shows a time sequence of the temperature, scalar dissipation rate, $\mathrm{CO}$ and $\mathrm{NO}$ mass fractions over mixture fraction, where the first four time values show different stages of the ignition process, while the latest corresponds to the time, when a stabile flame has been established in the LES. Additionally, a steady flamelet solution, which can be regarded as an attractor for the MRIF flamelet, is given as reference.

The plots in the lowest row show the decay of the scalar dissipation rate and additionally indicate the limits of the mixture fraction subfilter distribution. From the temperature profile plots in the upper row it can be seen that both, FWOT and MRIF flamelet results, show two distinct ignitions, first at rich conditions, then followed at nearly stoichiometric mixture. A cool flame, which is only present within the MRIF flamelet, transports heat and radicals from the most reactive mixture towards the rich side and thereby delays the ignition timing ( $\mathrm{OH}$ based) by approximately $45 \mu \mathrm{s}$ compared to the FWOT solution. However, even though the initiation of high-temperature chemistry is only weakly impacted, the cool flame increases the reactivity of rich mixtures compared to the no transport case significantly. As a result, higher temperatures and $\mathrm{CO}$ concentration can be observed in the MRIF flamelet for rich mixtures. Additionally, the maximum temperature and maximum $\mathrm{CO}$ mass fraction decrease due to turbulent tranport. At quasi-steady state, the maximum $\mathrm{CO}$ location is shifted towards richer mixtures in the MRIF flamelet and smaller CO concentrations are obtained at stoichiometric and lean conditions. A significant impact can also be observed in the formation of NO. After both, FWOT and MRIF flamelet have ignited, higher NO values are observed within the flamelet solution throughout the entire mixture fraction domain, while its maximum remains at stoichiometric conditions. Since, the FWOT shows slightly higher peak temperatures, the increased NO formation in the flamelet can be attributed to additional oxygen that is transported from the lean side into stoichiometric mixture. The heat, which is transported by turbulent mixing towards the lean side, enlarges the NO production zone, causing additional NO formation.

Overall, the comparison of MRIF and FWOT flamelet indicates that the pollutant formation process is strongly influenced by molecular mixing, which is enhanced by turbulent fluctuations. Thus, turbulence-chemistry interactions are important for predicting pollutants, while the ignition delay is only weakly affected. The increased reactivity of rich mixtures that is caused by radical and heat transport can also be expected to influence soot formation, which was, however, not included in this study.

\section{SUMMARY AND CONCLUSION}

A MRIF model for LES was presented and applied to the Spray A case of the ECN. The spray model imposes droplet information that have been extracted from a coupled LES/DNS of the nozzle-internal flow and primary breakup and no further parameter tuning of the Lagrangian spray 


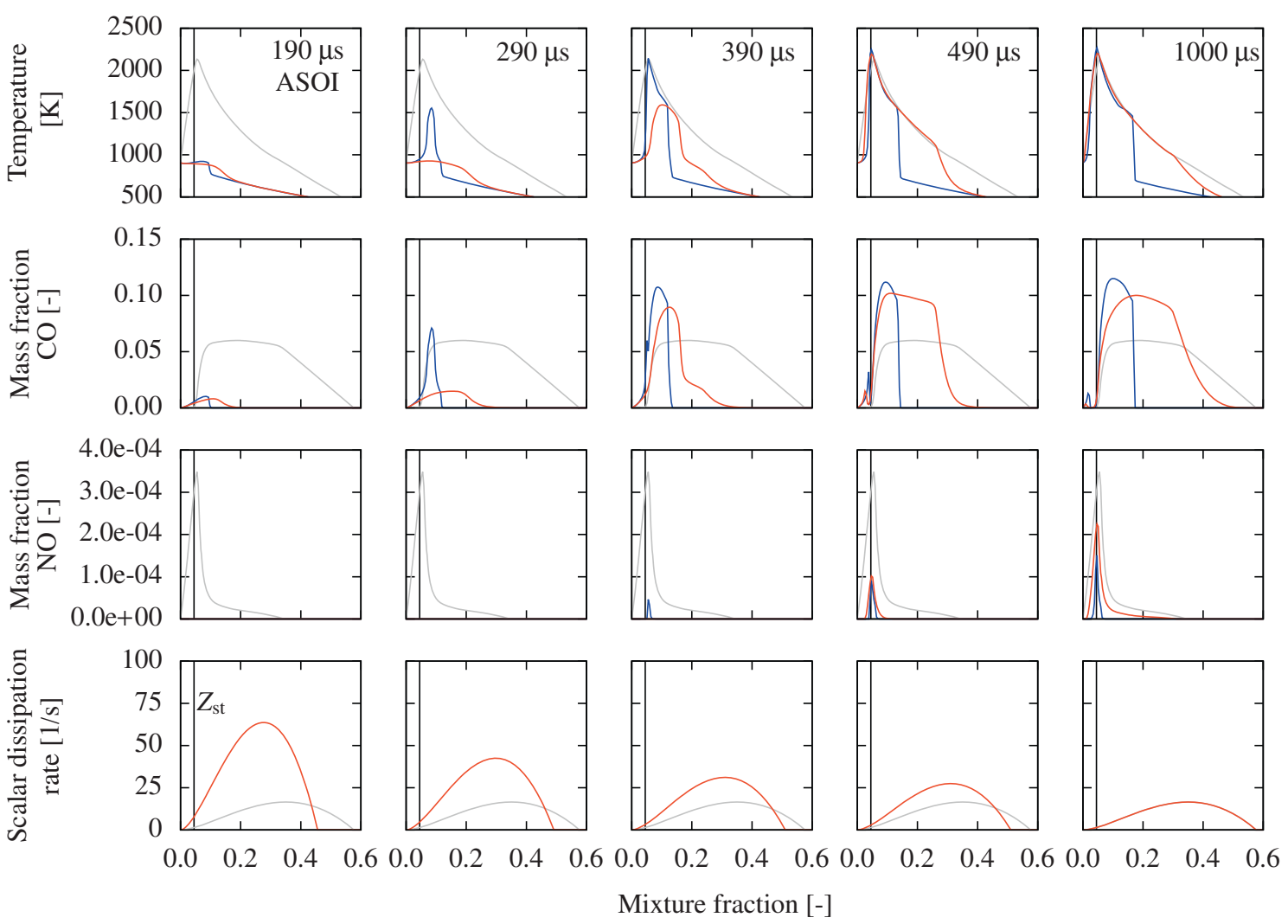

Figure 9

Temperature, scalar dissipation rate, CO, and NO mass fractions of the first MRIF flamelet (-), FWOT ( - ) and a steady state solution $(-)$ in mixture fraction space.

model was required. The spray model results were compared with experimental data in a non-reactive setup, showing very good agreement in terms of spray penetration, but simultaneously slightly underpredicting the fuel mass within the spray core. Nevertheless, in the reactive case, the combustion showed fairly good agreement with the characteristics of both, low temperature and high temperature combustion and hence, the MRIF model seems to capture the relevant physical processes of Diesel type combustion. The spray ignition process has been analyzed and showed strong diffusive transport in mixture fraction space, which corresponds to turbulent mixing in physical space. In the LES result, a cool flame was first initiated at approximately stoichiometric conditions and travelled towards rich mixtures, where a first ignition event was observed. The cool flame transports heat and radicals away from the most reactive mixture and thereby delays the ignition. Even though the difference in ignition timing between the LES and homogeneous reactor calculation is fairly small under the investigated conditions, turbulence-chemistry interactions have been found to affect both, the $\mathrm{CO}$ and the NO formation significantly. Since the cool flame increases the reactivity of rich zones, soot formation can also be expected to be strongly influenced by turbulence-chemistry interactions and should be investigated in future studies.

\section{ACKNOWLEDGMENTS}

The authors from RWTH Aachen University gratefully acknowledge funding from the Cluster of Excellence "Tailor-Made Fuels from Biomass" funded by the Excellence Initiative by the German Federal and State governments to promote science and research at German universities. This work was performed as a part of the GRK 1856 funded by the Deutsche Forschungsgemeinschaft (DFG, German Research Foundation). The authors gratefully acknowledge computing time granted for the project JHPC 18 by the JARA-HPC Vergabegremium and provided on the JARA-HPC Partition part of the supercomputer JUQUEEN [41] at Forschungszentrum Jülich. Additionally, the support of the Partnership for Advanced Computing in Europe (PRACE) provided under grant 2013092005 is gratefully acknowledged. S. Kang gratefully 
acknowledges the financial support from the National Research Council of Science \& Technology (NST) grant by the Korea government (MSIP) (No. CRC-15-07-KIER).

\section{REFERENCES}

1 Kalghatgi G.T. (2014). Developments in internal combustion engines and implications for combustion science and future transport fuels, Proc. Combust. Inst. 35, 101-115.

2 URL https://ecn.sandia.gov

3 Pope S.B. (1985) PDF methods for turbulent reactive flows, Prog. Energy Combust. Sci. 11, 2, 119-192.

4 Klimenko A.Y., Bilger R.W. (1999) Conditional moment closure for turbulent combustion, Prog. Energy Combust. Sci. 25, 6, 595-687.

5 Peters N. (1984) Laminar diffusion flamelet models in nonpremixed turbulent combustion, Prog. Energy Combust. Sci. 10, 3, 319-339.

6 Pitsch H., Wan Y.P., Peters N. (1995) Numerical investigation of soot formation and oxidation under Diesel engine conditions, SAE Technical Paper 952357.

7 Pitsch H., Chen M., Peters N. (1998) Unsteady flamelet modeling of turbulent hydrogen-air diffusion flames, Symp. Int. Combust. 27, 1, 1057-1064.

8 Barths H., Hasse C., Bikas G., Peters N. (2000) Simulation of combustion in direct injection Diesel engines using a Eulerian particle flamelet model, Proc. Combust. Inst. 28, 1, 1161-1168.

9 D’Errico G., Lucchini T., Contino F., Jangi M., Bai X.S. (2014) Comparison of well-mixed and multiple representative interactive flamelet approaches for Diesel spray combustion modelling, Combust. Theor. Model. 18, 1, 65-88.

10 Pei Y., Som S., Pomraning E., Senecal P.K., Skeen S.A., Manin J., Pickett L.M. (2015) Large eddy simulation of a reacting spray flame with multiple realizations under compression ignition engine conditions, Combust. Flame 162, 12, 4442-4455.

11 Blomberg C.K., Zeugin L., Pandurangi S.S., Bolla M., Boulouchos K., Wright Y.M. (2016) Modeling split injections of ECN "Spray A" using a conditional moment closure combustion model with RANS and LES, SAE Int. J. Engines 9, 2107-2119.

12 Wehrfritz A., Kaario O., Vuorinen V., Somers B. (2016) Large eddy simulation of $n$-dodecane spray flames using flamelet generated manifolds, Combust. Flame 167, 113-131.

13 Bekdemir C., Somers L.M.T., de Goey L.P.H., Tillou J., Angelberger C. (2013) Predicting Diesel combustion characteristics with large-eddy simulations including tabulated chemical kinetics, Proc. Combust. Inst. 34, 2, 3067-3074.

14 Germano M., Piomelli U., Moin P., Cabot W.H. (1991) A dynamic subgrid-scale eddy viscosity model, Phys. Fluids A: Fluid Dyn. 3, 7, 1760-1765.

15 Wehrfritz A., Vuorinen V., Kaario O., Larmi M. (2013) Large eddy simulation of high-velocity fuel sprays: studying mesh resolution and breakup model effects for spray A, Atomization Sprays 23, 5, 419-442.

16 Senecal P.K., Pomraning E., Xue Q., Som S., Banerjee S., Hu B., Liu K., Deur J.M. (2014) Large eddy simulation of vaporizing sprays considering multi-injection averaging and grid-convergent mesh resolution, J. Eng. Gas Turbines Power 136, 11, 111504 .
17 Bode M., Falkenstein T., Le Chenadec V., Kang S., Pitsch H., Arima T., Taniguchi H. (2015) A new Euler/Lagrange approach for multiphase simulations of a multi-hole GDI injector, $S A E$ Technical Paper. 2015-01-0949. SAE International.

18 Bode M., Davidovic M., Pitsch H. (2017) Multi-scale coupling for predictive injector simulations, Springer International Publishing, Cham, Switzerland, pp. 96-108.

19 Miller R.S., Harstad K., Bellan J. (1998) Evaluation of equilibrium and non-equilibrium evaporation models for many-droplet gas-liquid flow simulations, Int. J. Multiphase Flow 24, 6, 1025-1055.

20 Raman V., Pitsch H., Fox R.O. (2006) Eulerian transported probability density function sub-filter model for large-eddy simulations of turbulent combustion, Combust. Theor. Model. 10, 3, 439-458.

21 Doran E.M. (2011) A multi-dimensional flamelet model for ignition in multi-feed combustion systems, PhD Thesis, Stanford University, Stanford, CA.

22 Pitsch H., Steiner H. (2000) Scalar mixing and dissipation rate in large-eddy simulations of non-premixed turbulent combustion, Proc. Combust. Inst. 28, 1, 41-49.

23 Liu X.-D., Osher S., Chan T. (1994) Weighted essentially nonoscillatory schemes, J. Comput. Phys. 115, 1, 200-212.

24 Desjardins O., Blanquart G., Balarac G., Pitsch H. (2008) High order conservative finite difference scheme for variable density low Mach number turbulent flows, J. Comput. Phys. 227, 15, 7125-7159.

25 Mittal V., Kang S., Doran E., Cook D., Pitsch H. (2014) LES of gas exchange in IC engines, Oil Gas Sci. Technol. - Rev. IFP 69, 1, 29-40.

26 Dukowicz J.K. (1980) A particle-fluid numerical model for liquid sprays, J. Comput. Phys. 35, 2, 229-253.

27 Apte S.V., Mahesh K., Lundgren T. (2008) Accounting for finite-size effects in simulations of disperse particle-laden flows, Int. J. Multiphase Flow 34, 3, 260-271.

28 Pickett L.M., Genzale C.L., Bruneaux G., Malbec L.-M., Hermant L., Christiansen C., Schramm J. (2010) Comparison of Diesel spray combustion in different hightemperature, high-pressure facilities, SAE Int. J. Engines 3, 156-181.

29 Pickett L.M., Manin J., Genzale C.L., Siebers D.L., Musculus M.P.B., Idicheria C.A. (2011) Relationship between Diesel fuel spray vapor penetration/dispersion and local fuel mixture fraction, SAE Int. J. Engines 4, 764-799.

30 Skeen S.A., Manin J., Pickett L.M. (2015) Simultaneous formaldehyde PLIF and high-speed schlieren imaging for ignition visualization in high-pressure spray flames, Proc. Combust. Inst. 35, 3, 3167-3174.

31 Knudsen E., Shashank, Pitsch H. (2015) Modeling partially premixed combustion behavior in multiphase LES, Combust. Flame 162, 1, 159-180.

32 Ham F., Apte S., Iaccarino G., Wu X., Herrmann M., Constantinescu G., Mahesh K., Moin P. (2003) Unstructured LES of reacting multiphase flows in realistic gas turbine combustors, in CTR annual research briefs, pp. 139-160

33 Narayanaswamy K., Pepiot P., Pitsch H. (2014) A chemical mechanism for low to high temperature oxidation of $n$-dodecane as a component of transportation fuel surrogates, Combust. Flame 161, 4, 866-884. 
34 Vasu S.S., Davidson D.F., Hong Z., Vasudevan V., Hanson R. K. (2009) $N$-dodecane oxidation at high-pressures: Measurements of ignition delay times and $\mathrm{OH}$ concentration timehistories, Proc. Combust. Inst. 32, 1, 173-180.

35 Pepiot-Desjardins P., Pitsch H. (2008) An efficient error propagation-based reduction method for large chemical kinetic mechanisms, Combust. Flame 154, 67-81.

36 Pepiot-Desjardins P., Pitsch H. (2008) An automatic chemical lumping method for the reduction of large chemical kinetic mechanisms, Combust. Theor. Model. 12, 6, 1089-1108.

37 Frenklach M. (1984) Systematic optimization of a detailed kinetic model using a methane ignition example, Combust. Flame 58, 1, 69-72.

38 Lamoureux N., Desgroux P., El Bakali A., Pauwels J.F. (2010) Experimental and numerical study of the role of $\mathrm{NCN}$ in prompt-NO formation in low-pressure $\mathrm{CH}_{4}-\mathrm{O}_{2}-\mathrm{N}_{2}$ and $\mathrm{C}_{2} \mathrm{H}_{2}-\mathrm{O}_{2}-\mathrm{N}_{2}$ flames, Combust. Flame 157, 10, 1929-1941.
39 Narayanaswamy K., Blanquart G., Pitsch H. (2010) A consistent chemical mechanism for oxidation of substituted aromatic species, Combust. Flame 157, 10, 1879-1898.

40 Idicheria C.A., Pickett L.M. (2007) Quantitative mixing measurements in a vaporizing Diesel spray by Rayleigh imaging, SAE Technical Paper. 2007-01-0647. SAE International.

41 Jülich Supercomputing Centre (2015) JUQUEEN: IBM Blue Gene/Q supercomputer system at the Jülich supercomputing centre, Journal of Large-Scale Research Facilities 1, 1-5.

Manuscript submitted in February 2017 Manuscript accepted in June 2017 Published online in October 2017 